\title{
Ageísmo, políticas públicas voltadas para população idosa e participação social
}

\author{
Ageism, public policies for the elderly and social participation
}

Ageísmo, políticas públicas para los ancianos y participación social

Vitor Hugo Sales Ferreira ${ }^{1 *}$, Luiza Rosa Bezerra Leãoํㄹ Andréa Mathes Faustino1.

\section{RESUMO}

Objetivo: Apresentar o processo histórico em que foi pautada a construção das políticas públicas ligadas ao envelhecimento no Brasil, levando-se em consideração a importância da participação social e do fenômeno do ageísmo na sociedade brasileira. Revisão Bibliográfica: $O$ processo de evolução histórica sobre o envelhecimento populacional no Brasil, têm como marcos a criação da Sociedade Brasileira de Geriatria e Gerontologia e os grupos de convivências voltados para idosos do Serviço Social do Comércio nos anos de 1960, e estes se desenvolvem até grandes marcos históricos como a criação do Conselho Nacional da Pessoa Idosa e a Política Nacional do Idoso a partir dos anos 2000. Estas foram parte das diretrizes utilizadas para iniciar parte da desconstrução do padrão ageísta de construções governamentais e sociais para o envelhecimento. Considerações finais: $O$ Brasil foi pioneiro no que se refere ao pensar no envelhecimento populacional, entretanto, sua implementação não ocorre de forma simultânea à transição demográfica, principalmente no que se refere estudos e implementações voltadas ao ageísmo.

Palavras-chave: Ageísmo, Política pública, Idoso.

\begin{abstract}
Objective: To present the historical process that guided the construction of public policies related to aging in Brazil, in consideration of social participation and the phenomenon of ageism in Brazilian society. Bibliographic Review: The process of historical evolution about population aging in Brazil, have one of your milestones the creation of the Brazilian Society of Geriatrics and Gerontology and the elderly social groups of Social Service in the years 1960s, and these develop into major historical milestones such as the creation of the National Elderly Council and the National Elderly Policy from the 2000s. These were part of the guidelines to start the deconstruction process of the ageist pattern of governmental and social constructs for aging. Final Considerations: Brazil was a pioneer in thinking about population aging, however, its implementation does not occur simultaneously to the demographic transition, especially regarding studies and implementations aimed at ageism.
\end{abstract}

Keywords: Ageism, Public policy, Aged.

\section{RESUMEN}

Objetivo: Presentar el proceso histórico que ha sido pautada la construcción de políticas públicas relacionadas con el envejecimiento poblacional en Brasil, teniendo en cuenta la importancia de la participación social y fenómeno del ageísmo en la sociedad brasileña. Revisión bibliográfica: El proceso de evolución histórica del envejecimiento de la población pensante brasileña, tiene uno de sus hitos en la creación de la

${ }^{1}$ Centro de Estudos Avançados Multidisciplinares (CEAM), Universidade de Brasília (UnB), Brasília-DF.

*E-mail: vitorhugosalesferreira@gmail.com 
Sociedad Brasileña de Geriatría y Gerontología y los grupos sociales mayores de Servicio Social en los años 1960, y estos se ha convertido en hitos históricos importantes como la creación del Consejo Nacional de Ancianos y la Política Nacional de Ancianos a partir de la década de 2000. Estas fueron parte de las pautas para que ha empezado como parte de la deconstrucción de la norma de la construcción socialista del gobierno y construcciones sociales para el envejecimiento. Consideraciones Finales: Brasil fue pionero en pensar sobre el envejecimiento de la población, sin embargo, su implementación no ocurre simultáneamente a la transición demográfica, especialmente en lo que respecta a los estudios e implementaciones dirigidos al envejecimiento.

Palabras clave: Ageísmo, Políticas públicas, Ancianos.

\section{INTRODUÇÃO}

Envelhecer ao longo da história humana já foi privilégio para poucos. A realidade, atualmente vem se alterando dramaticamente desde a segunda metade do século XX. A partir desse período, envelhecer é cada vez mais parte de um processo natural esperado para muitas populações ao redor do globo: a expectativa de vida aumentou em 20 anos em todo o mundo desde 1950 a 2002. Dados da Organização das Nações Unidas (ONU) projetam estimativa de 2 bilhões de pessoas idosas no mundo em 2050, sendo idosa toda pessoa com 60 anos ou mais, segundo a própria ONU (SOUZA FDS, 2010).

A mudança na estrutura demográfica dos diversos países desenvolvidos e, mais recentemente, dos países em desenvolvimento, é marcada pela associação entre diminuição das taxas de fecundidade e aumento da expectativa de vida através de melhorias no acesso a ações e serviços de saúde integrais e resolutivos, além de investimentos em infraestrutura, saneamento básico, acesso à educação, dentre outros Determinantes Sociais em Saúde (DSS).

Todos esses fatores foram visibilizados em um momento da história que se entendia que os estados nacionais deveriam ser os motores promotores de ações para melhoria da economia e da qualidade de vida da população, e a visão Keynesiana de estado em que predominam políticas de bem-estar social estava em foco (BORDE E, et al., 2015; FELIX JS, 2009).

Em resumo, segundo a Secretaria de Previdência do Ministério da Fazenda (BRASIL, 2013), constatouse que, nesse mesmo ano, uma em cada dez pessoas ultrapassou a idade de 60 anos no Brasil, e a estimativa para 2060 apontou ser ainda mais alarmante, em que uma em cada três pessoas ultrapassaria essa mesma faixa etária, assim também afirmaram outros órgãos, como o Instituto Brasileiro de Geografia e Estatística (IBGE), que em 2010 que estimou que uma em cada quatro pessoas atingiu idade igual ou superior à 65 anos.

Assim, entende-se que a melhor forma de lidar com a inversão da pirâmide etária brasileira, seja a partir da preocupação acerca das políticas públicas de caráter preventivo e de promoção à saúde de cidadão brasileiro e de toda pessoa dentro do território nacional (IBGE, 2010).

A transição demográfica de um país de jovens para um país envelhecido, contudo, ocorre a passos largos no Brasil. Vários estudiosos concordam que o país alcançou em uma geração, apenas, o processo que a Europa realizou em aproximadamente cem anos (CAMACHO LF, et al., 2010; WILLIG MH, et al., 2012; ANDRADE LM et al., 2013; SANTOS NF, SILVA MRF, 2013).

De acordo com o Estatuto do Idoso, toda pessoa com mais 60 anos é considerada idosa, o que, segundo os estudos demográficos de 2010, representa $11 \%$ da população brasileira, ou 20 milhões de pessoas idosas (BRASIL, 2003). A partir de 2020, espera-se que o Brasil se torne a sexta nação mais envelhecida do mundo, com população estimada em 32 milhões de pessoas (ANDRADE LM, et al., 2013; LEÃO LRB, et al., 2018).

A grande velocidade da transição demográfica experimentada no Brasil em comparação com países europeus incutiu caráter de urgência no desenvolvimento de políticas públicas para lidar com 0 envelhecimento da população. As crises econômicas, financeiras e políticas sucessivas que o país atravessa 
desde o início do século $X X$ até hoje geram suas próprias demandas sobre as possibilidades de se construir políticas sociais. Os diversos estados europeus puderam desenvolver suas políticas no cenário característico de países desenvolvidos (BORDE E, et al., 2015; FELIX JS, 2009).

A velhice é, portanto, um processo natural, mas tão plural quanto as diversas culturas humanas existentes e ainda diverso de acordo com a experiência vivida por cada indivíduo. Processo esse que supera em muito alterações fisiológicas próprias de desgastes do organismo ao longo da vida e da perda de funcionalidades.

Em termos biológicos, avalia-se o processo de envelhecimento saudável (senescência) através da mensuração da capacidade funcional da pessoa para Atividades de Vida Diária (AVDs), que são atividades como, por exemplo, tomar banho sem auxílio, discar números de telefone ou vestir-se (MUNIZ EA et al., 2016). Outras alterações, contudo, acompanham o envelhecimento, com mudanças em todas as escalas da vida da pessoa: a forma como a pessoa se relaciona consigo própria e com a sociedade que a rodeia (SANTOS NF, SILVA MRF, 2013).

Organismos nacionais e internacionais vêm discutindo aspectos de previdência social e sistemas de saúde, diretamente impactados pelo envelhecimento populacional. Muitas das estratégias adotadas envolvem trabalhar o envelhecimento ativo, a fim de, como foi citado por alguns autores "compensar as despesas que o estado dispensará a esse grupo populacional" (SOUZA FDS, 2010).

Campo de estudo emancipado da ciência política por Harold Lasswell, políticas públicas (policy) compõemse da formalização de normas e regras oriundas de processos informais de disputa de poder e negociação por parte dos diversos atores sociais, ou seja, são o processo do estado trabalhando na sociedade, o gerúndio.

Elas são adotadas em contextos em que há relações de poder e objetivam garantir interesses considerados públicos, mediante olhares diversos e até mesmo antagônicos, como o campo complexo, interdisciplinar e amadurecido que ele o policy é (BULLOSA RF, 2015).

A construção de políticas públicas para pessoas idosas perpassa todas essas características anteriormente citadas, além de somar ainda o preconceito etário envolvido na forma como a população encara o envelhecimento. A esse preconceito, dá-se o nome ageísmo. Percebe-se, portanto, diversas vulnerabilidades na construção de políticas públicas do envelhecimento (CAMACHO LF, et al., 2010; SANTOS NF, SILVA MRF, 2013).

Atribuído ao desenvolvimento capitalista e à sua lógica de validação social das pessoas por meio da capacidade delas de trabalhar e serem produtivas, o ageísmo é o nome que denomina discriminação social sistemática voltada especificamente contra as pessoas idosas. O medo da morte é outro fator predisponente ao preconceito contra os idosos, vistos como símbolo de adoecimento, enfraquecimento e morte. Alguns autores, consideram o fenômeno do ageísmo uma demonstração explícita de um medo irracional da morte. Acrescente-se a isso o culto à juventude, historicamente exacerbado no Brasil, para construir o imaginário social nem sempre explícito de grande aversão ao envelhecimento (SANTOS NF, SILVA MRF, 2013; FELIX JS, 2009).

Conferem-se aspectos pejorativos, estereotipias. Os meios de comunicação de massa, como emissoras de televisão e de rádio, são considerados os principais meios de propagação desses estereótipos pejorativos na contemporaneidade. No mundo ocidental, o ageísmo é a terceira forma de discriminação mais prevalente, atrás apenas do racismo e do sexismo.

Reconhecer a pluralidade de experiências de envelhecer associadas a iniciativas individuais, comunitárias e governamentais de manutenção da inclusão social e combate ao isolamento social dos idosos são encorajadas a fim de evitar esse imaginário coletivo de miséria do processo de envelhecimento: algo repleto de doenças, de dependência e de passividade, devendo, portanto, ser evitado (SANTOS NF, SILVA MRF, 2013).

Desta feita, o objetivo da presente revisão crítica é apresentar o processo histórico em que foi pautada a construção das políticas públicas ligadas ao envelhecimento no Brasil, levando-se em consideração a importância da participação social e do fenômeno do ageísmo na sociedade brasileira. 


\section{REVISÃO BIBLIOGRÁFICA}

\section{Iniciativas e políticas de amparo ao idoso: dos primeiros movimentos sociais até o conceito de envelhecimento saudável}

Quaisquer trabalhos que se pautem no processo histórico de iniciativas e de políticas de amparo ao idoso a fim de compreender as políticas públicas atuais precisam mencionar momentos da história anteriores até mesmo ao atual regime político democrático no Brasil.

Os primeiros movimentos sociais do século XX ocorreram nos anos de 1917, por trabalhadores que buscavam direito a melhores condições de trabalho, sendo toda a primeira metade do século XX pautada na busca por direitos trabalhistas. A lógica da produção, típica da Era Vargas, servia como motor para construção dessas primeiras políticas públicas no país (JOSÉ et al., 2015)

Previdência foi uma pauta de destaque durante o estado Novo e, com ela, o olhar sobre a pequena parcela da população idosa. A sociedade civil foi fundamental para que pautas ligadas ao envelhecimento ganhassem importância e visibilidade na agenda do estado. Inicialmente os principais atores sociais envolvidos foram os membros da Sociedade Brasileira de Geriatria e Gerontologia (SBGG), criada em 1961 e os grupos de convivência do Serviço Social do Comércio (SESC).

A preocupação basilar era com o desamparo e a solidão que viriam a atingir os idosos com a aposentadoria. As demandas levantadas, entretanto, esbarravam no desinteresse do aparelho estatal. É da década de 60 também o início da atuação da União dos Aposentados e Pensionistas, provavelmente a mais relevante e direta forma de atuação dos próprios idosos na arena política (WILLIG MH et al., 2012; FERLICE DS, SOUZA AL, 2010; FERLICE DS, SOUZA AL, 2010).

Em 1975, durante o regime militar, foi criado o Ministério da Previdência e Assistência Social (MPAS), momento em que se iniciou a prestação de assistência social específica para a população idosa em áreas referentes a renda, saúde e prevenção de asilamento. Em 1976 foram redigidas as primeiras Diretrizes para uma Política Nacional da Terceira Idade, com normas pautadas na assistência de idosos fisicamente dependentes e socialmente vulneráveis (WILLIG MH et al., 2012)

A década de 80 no Brasil, foi marcada por intensas mobilizações sociais: pelas Diretas-já, por direitos sociais, por liberdade de expressão, dentre outros. Em 1982 ocorreu a I Assembleia Mundial do Envelhecimento, evento da Organização das Nações Unidas (ONU) em Viena-Áustria, onde se cunhou o conceito de envelhecimento saudável no Plano de Ação para o Envelhecimento, sendo a primeira iniciativa de atores estatais a fim de trabalhar questões relativas à população idosa de forma intersetorial, ou seja: para além da esfera econômica.

No Brasil, dentre as políticas públicas ligadas à população idosa, cita-se a Lei $8.842 / 84$, que trata da Política Nacional do Idoso, cria o Conselho Nacional do Idoso e dá outras providências, tendo ela sido fortemente pela Assembleia da ONU, em Viena (WILLIG MH et al., 2012; FERLICE DS, SOUZA AL, 2010).

\section{A Constituição Cidadã e a reorientação das políticas públicas ligadas ao envelhecimento}

Envelhecimento saudável, contudo, foi um conceito incorporado apenas na Constituição Federal de 1988, apelidada de Constituição Cidadã. Somente após a mencionada Assembleia, as políticas públicas brasileiras ampliaram seus objetivos para além de assistir aos idosos vulneráveis (WILLIG MH et al., 2012; SANTOS NF, SILVA MRF, 2013).

A partir da Constituição Federal de 1988, o estado brasileiro, com seus artigos 196 a 200, assumiu a responsabilidade de prover saúde para toda a população por meio de políticas econômicas e sociais, o que marcou abrupta reorientação da forma com que o estado assistia ao povo: pela primeira vez, a lógica varguista de ser trabalhador formal não é fator preponderante para ter acesso à saúde pública (BRASIL, 1988; JOSÉ $\mathrm{M}$, et al., 2015).

O direito à saúde foi ainda reafirmado através da Lei 8.080/1990, em que foi criado o Sistema Único de Saúde (SUS) e a participação social continuou sendo reafirmada como componente fundamental para a construção de sistema de saúde integral, humano e resolutivo, de acordo com as especificidades da 
população brasileira como um todo, mas também levando em consideração vulnerabilidades sociais, econômicas e de estado de saúde (BRASIL, 1990; WILLIG MH, et al., 2012).

Em 1994, origina-se a Lei n 8.842, de 4 de janeiro de 1994. Dispõe sobre a Política Nacional do Idoso, cria o Conselho Nacional do Idoso (CDI) e dá outras providências, sendo regulamentada pelo Decreto 1948/96.

Alinhada com o espírito da época de políticas públicas intersetoriais, como a política pública do SUS, a Lei 8.842/94 ampliou ainda mais a articulação das áreas a serem integradas na formulação de políticas públicas para a população idosa, elencando além dos campos da Seguridade Social (Previdência, Assistência Social e Saúde), ainda: Cultura, Trabalho, Planejamento, dentre outros. É esse arcabouço jurídico que propiciou, em 1996, o Decreto 1.948/96 a estabelecer a Política Nacional do Idoso (PNI), definindo a Seguridade Social como articuladora central da PNI (WILLIG MH, et al., 2012).

\section{O século XX: desafios para a consolidação da visão ampliada de envelhecimento}

Os anos 2000, já com parcela considerável de idosos presente na população, foram decisivos para as políticas públicas ligadas ao envelhecimento e à população idosa. Nesse período, estão o Decreto 4.227/2002, que regulamenta o Conselho Nacional dos Direitos dos Idosos (CNDI), sendo seu grande avanço a proposta da formulação de políticas públicas para os idosos com ampla e ativa participação da população idosa brasileira.

Tal decreto veio a ser revogado, revisto e ampliado pelo Decreto 5.109/2004. Este último revogado e atualizado com o Decreto 9.893/2019, em que uma das principais mudanças foi a alteração da vinculação do Conselho Nacional ao anteriormente chamado Ministério dos Direitos Humanos e recentemente renomeado para Ministério da Mulher, da Família e dos Direitos Humanos (MMFDH), restringindo, portanto, a efetiva atuação do Conselho, além da anterior prerrogativa de ampla participação popular (BRASIL, 2019).

Ainda do início dos anos 2000, a Lei 10.741/03, que estabelece o Estatuto do Idoso e representou um verdadeiro marco para a população idosa brasileira, e a Portaria 2.528/06 que cria a Política Nacional de Saúde da Pessoa Idosa (PNSPI) revogando e ampliando a portaria 1.395/99, que tratava da Política Nacional do Idoso (PNI).

A PNSPI de 2006 incorporou de fato o conceito de envelhecimento ativo preconizado pela Organização Mundial de Saúde, em 2002, além combater o ageísmo, estimular a participação social dos idosos na comunidade e na formulação das políticas públicas (BRASIL, 2003; BRASIL, 2006).

Percebe-se, sobretudo com a portaria 2.520/06 que a histórica visão do envelhecimento como um processo repleto de adoecimento, vulnerabilidade e solidão para se pensar política pública havia sido superada.

$\mathrm{O}$ arcabouço legal brasileiro vem assim sendo considerado complexo, completo e abrangente para lidar com as necessidades de parcela cada vez maior do país, atribuindo, especialmente a partir dos anos 2000, novo olhar, mais humano e repleto de potencialidades para o envelhecimento (WILLIG MH, et al., 2012; ROZENDO AS, 2016).

\section{A realidade dos idosos brasileiros $x$ o arcabouço legal: avanços e desafios}

A vivência prática de muitos idosos, contudo, nem sempre acompanham as políticas públicas e o que se percebe nos relatos de diversos autores são grandes e variados processos de violência e de exclusão social de idosos por parte de uma sociedade ainda incapaz de lidar não só com o futuro da população em idade ativa, mas também com o próprio presente, de nação envelhecida, diversa, plural que são as pessoas idosas no Brasil (SANTOS NF, SILVA MRF, 2013; CAMACHO LF, et al., 2010; WILLIG MH, et al., 2012; ANDRADE LM, et al., 2013; FERLICE DS e SOUZA AL, 2010).

Considerando as dimensões continentais do Brasil, há consequências decorrentes da diversidade geográfica e espacial, especialmente em relação às políticas públicas para a terceira idade. Ainda que nas últimas décadas, tenham sido criadas e consolidadas várias políticas para atender as demandas dessa população. 
O Sistema Único de Saúde (SUS), a maior política de bem-estar social brasileira, a Política Nacional de Saúde da Pessoa Idosa (PNSPI), dentre outras, são algumas provas de que com engajamento, financiamento, fiscalização e interesse em manutenção da continuidade dos processos, há viabilidade para formulação e manutenção de ações públicas em prol do bem-estar das pessoas residentes em território nacional (FELIX JS, 2009; FERLICE DS, SOUZA AL, 2010).

O Plano de Madri, de 2002, um documento amplo com 35 objetivos dirigidos aos governos nacionais em parceria com a sociedade e a iniciativa privada, foi tão marcante como ponto de ruptura das antigas políticas públicas ageístas quanto a I Assembleia Nacional do Envelhecimento da ONU em Viena-Áustria, apesar de falhar no mesmo ponto: desconsideração pelas fontes de recursos para cumprimento das metas estabelecidas.

Dentre os três maiores avanços, cita-se: 1) enfatizar a participação da comunidade, em especial, da comunidade de idosos, na formulação das políticas públicas de saúde, o que ainda hoje se mostra um desafio a ser cumprido; 2) estímulo ao envelhecimento saudável e ativo, outro desafio ainda muito presente na realidade nacional e 3) criação de ambiente favorável à qualidade de vida na terceira idade, o que parece ser mais dificultoso ainda de ser realizado sem a adequada intervenção do estado (FELIX JS, 2009; WILLIG MH, et al., 2012; ; FERLICE DS, SOUZA AL, 2010).

\section{O imaginário coletivo atual de descrença no papel do estado: consequências para as políticas públicas que tratam do envelhecimento}

Vive-se, atualmente, momentos de descrença da importância do estado, não só no Brasil, mas em todo o mundo. O modelo de estado promotor de ações de infraestrutura e de políticas de bem-estar social para atingir desenvolvimento nacional, como era proposto por Keynes e amplamente aceito em toda a América Latina até a década de 80, hoje é considerado um modelo político-econômico desgastado.

Modelos alternativos de estado vem nascendo e se multiplicando por toda a América, sendo praticamente impossível afirmar o que é mais adequado para cada realidade nacional. Entretanto, é consenso entre diversas esferas de profissionais das áreas de políticas públicas, de gestão pública, de ciência política, de economia, dentre outros, que seguridade social é uma necessidade para aplacar as iniquidades sociais de um dos países mais desiguais do mundo (FELIX SJ, 2009).

A proporção que tais políticas públicas devem ocupar na agenda e nos gastos estatais podem variar de acordo com o modelo político e econômico de estado adotado em governos diversos, contudo não há como pensar qualidade de vida na terceira idade, para o que se tornará em breve a maior parcela da população, sem o estado estar diretamente envolvido em tal discussão.

Os planos de Viena-Áustria e de Madri têm favorecido o surgimento de excelentes políticas públicas inclusivas e positivas quanto ao fenômeno do envelhecimento populacional. Interromper esse fluxo de progresso e de participação social juridicamente consolidada, contudo, se constituiria em um retrocesso.

É imprescindível criar ou desenvolver estratégias de aproximação da população na construção e reforma das políticas públicas já existentes, a fim de dar a elas dinamicidade o mais próximo possível daquela orgânica à nossa grande velocidade atual de transformações demográficas, sociais e econômicas. Repensar a regulação social na ação pública ligada ao envelhecimento é preciso.

\section{CONSIDERAÇÕES FINAIS}

A rápida transição demográfica experimentada no Brasil em comparação com países europeus faz com que haja em caráter de urgência o desenvolvimento de políticas públicas para lidar com o envelhecimento da população. Crises econômicas, financeiras e políticas sucessivas que o país atravessou desde o início do século XX repercutem até o presente momento. Tentativas foram realizadas para ampliar o olhar sobre 0 envelhecimento, sob a ótica de outros atores sociais. A Lei que instituiu o Conselho do Idoso foi um exemplo disto. A alteração com o decreto de 2019 reduziu seu poder deliberativo. Além de todo esse cenário, somase o ageísmo da sociedade brasileira e percebe-se, portanto, diversas vulnerabilidades na construção de políticas públicas que pensem o envelhecimento. 


\section{REFERÊNCIAS}

1. ANDRADE LM, et al. Políticas públicas para pessoas idosas no Brasil: uma revisão integrativa. Public policies for the elderly in Brazil: an integrative review. Ciência \& Saúde Coletiva, 2013, 18(12): 3543-3552.

2. BORDE E, et al. Uma análise crítica da abordagem dos Determinantes Sociais da Saúde a partir da medicina social e saúde coletiva latino-americana. Saúde em Debate, 2015, 39(106): 841-854.

3. BRASIL. MINISTÉRIO DA SAÚDE. Dispõe sobre as condições para a promoção, proteção e recuperação da saúde, a organização e o funcionamento dos serviços correspondentes e dá outras providências. Diário Oficial da União, 1990: 1-13.

4. BRASIL. MINISTÉRIO DA SAÚDE. Estatuto do Idoso 3a edição $2^{a}$ reimpressão. 2003.

5. BRASIL. MINISTÉRIO DA SAÚDE. Portaria GM nº 2.528 de 19 de outubro de 2006: Política Nacional de Saúde da Pessoa Idosa. Brasília. DF. Diário Oficial da União, 2006: 1-13.

6. BRASIL. PRESIDÉNCIA DA REPÚBLICA. Decreto ${ }^{\circ} 4.227$, de 13 de maio de 2002. Cria o Conselho Nacional dos Direitos do Idoso - CNDI, e dá outras providências. 2002: 12-13.

7. BRASIL. PRESIDÊNCIA DA REPÚBLICA. Nota pública do conselho nacional dos direitos da pessoa idosa - CNDI sobre a publicação do decreto 9.893/2019, 2019: 1-3.

8. BRASIL. Título VIII, Capítulo II, Seção II, Artigos de 196 a 200, da Saúde. BRASIL, Legislação Federal, Leis Federais n 8.080. Constituição Federal, 1988: 1-2.

9. CAMACHO LF, et al. Políticas públicas para a saúde do idoso: revisão sistemática. Revista Brasileira de Enfermagem, 2010, 63(2):279-248.

10. FELIX JS. Economia da longevidade: o envelhecimento da população brasileira e as políticas públicas para os idosos (Mestrado em Economia Política). Pontifícia Universidade Católica de São Paulo (PUC-SP), São Paulo, SP, 2009: 107.

11. FERLICE DS, SOUZA AL. Diretrizes internacionais e politicas para os idosos no Brasil: a ideologia do envelhecimento ativo. Revista de Políticas Públicas, 2010, 14(1): 85-94.

12. JOSÉ M, et al. Envelhecimento da população e as políticas públicas de saúde. Revista do Instituto de Políticas Públicas de Marília, 2015:26-34.

13. LEÃO LRB, et al. Functional capacity and resilience in hospitalized older adults. Journal of Nursing UFPE on line, 2018, 12(6):1500-1506.

14. MUNIZ EA, et al. Desempenho nas atividades básicas da vida diária de idosos em Atenção Domiciliar na Estratégia Saúde da Família. Revista Kairós Gerontologia, 2016, 19(2):133-146.

15. ROTHER ET. Revisão sistemática X revisão narrativa. Acta Paulista de Enfermagem, 2007, 20(2):v-vi.

16. ROZENDO AS. Ageísmo: um estudo com grupos de Terceira Idade. Revista Kairós: Gerontologia, 2016, 19(3):7989.

17. SANTOS NF, SILVA MRB. As políticas públicas voltadas ao idoso: melhoria da qualidade de vida ou reprivatização da velhice. Revista FSA. 2013, 20(2):358-371.

18. SILVA FD, SOUZA AL. Diretrizes internacionais e políticas para os idosos no brasil: a ideologia do envelhecimento ativo. Revista de Políticas Públicas, 2010, 14(1):85-94.

19. WILLIG MH, et al. A trajetória das políticas públicas do idoso no Brasil: breve análise. Cogitare enfermagem, 2012 , $17(3): 574-577$. 